\title{
Utilisation de la télédétection pour l'identification des parcelles irriguées
}

\author{
Use of teledetection for identification \\ of fields under irrigation
}

Guy Roman

Agence de Bassin « Loire-Bretagne», Orléans
Paul Almayrac

SETEC International, Vitrolles
Les sécheresses successives de ces dernières années et tout particulièrement 1976, ont rappelé au grand public la précarité de nos ressources en eau même en France au $\mathrm{XX}^{\mathrm{e}}$ siècle.

Les solutions provisoires adoptées en 1976 pour palier les effets de la pénurie ont montré que la lutte contre la sécheresse ne s'improvisait pas tant au niveau des collectivités que des irrigants.

Si les limitations, voire les interdictions de prélèvements, ont permis en dernier recours de gérer la pénurie, elles ne sauraient constituer une solution compatible avec les nécessités modernes d'une maîtrise de l'eau. Seule une véritable gestion de cette ressource s'appuyant sur une connaissance effective de la demande en eau, de sa localisation et de ses caractéristiques d'une part, et de la Trésorerie Eau d'autre part, est de nature à prévoir et prévenir par les moyens adéquats, les déséquilibres ressources-besoins.

En ce qui concerne la demande en eau dans le Bassin "Loire-Bretagne", il apparaît que si les prélèvements des réseaux de distribution d'eau aux collectivités sont connus avec une bonne précision $(>90 \%)$ tout comme ceux des industriels, il n'en est pas de même pour les agriculteurs pratiquant l'irrigation.

Or l'irrigation de complément joue dans le Bassin "Loire-Bretagne" un rôle très important dans l'équilibre Ressources-Besoins :

- Par sa pratique sur un court laps de temps (15 juin 15 août)

- Par la variabilité des apports nécessaires (de zéro à $2500 \mathrm{~m}^{3} /$ ha d'autant plus importants que l'année est plus sèche)

- Par la valeur des débits appelés $2,5 \mathrm{~m}^{3} / \mathrm{h} / \mathrm{ha}$.

De ce fait, l'Agence de Bassin a été amenée à rechercher, localiser et identifier les parcelles irriguées.

La circonscription de bassin "Loire-Bretagne" couvre une superficie importante $\left(155000 \mathrm{~km}^{2}\right)$ soit près du tiers du territoire national dont $110000 \mathrm{~km}^{2}$ sont utilisés pour l'Agriculture.

L'irrigation qui a commencé à se développer à l'initiative individuelle en "Loire-Bretagne" en 1962 représentait d'après les données du Recensement Général de l'Agriculture, près de 105000 ha en 1970 , localisés principalement dans les régions Centre et Pays de la Loire.

Le problème posé est donc de sélectionner rapidement à un coût acceptable et dans des délais très limités, $0,6 \%$ de la superficie du Bassin.

La méthode envisagée doit permettre d'identifier les parcelles irriguées quels que soient :

- la culture (maïs, betteraves, légumes de plein champs, vergers, terrains maraîchers) ;

- le type de sol (profond, superficiel);

- le mode d'irrigation (ruissellement, goutte à goutte, aspersion) ;

- le type de matériel (asperseurs, canon, pivot système).

La note ci-après présente successivement les fondements de la méthode, l'opération "Télédétection Irrigants 1976", les résultats obtenus, et dégage les perspectives d'amélioration et de recherche sur le thème de la connaissance de l'irrigation par télédétection.

Fondements et principes de la détection de l'irrigation des cultures

Le principe de la méthode consiste à analyser et interpréter des photographies aériennes obtenues à partir d'émulsions infra-rouge et de filtres adaptés, mettant en évidence l'irrigation. En effet, la mise en évidence de l'irrigation sur des photographies repose sur deux types de critères qui sont les uns géométriques et les autres radiométriques. 


\section{Critères géométriques}

Les critères géométriques constituent des indices apparents liés aux équipements et aux matériels utilisés pour l'irrigation :

- traces circulaires des asperseurs fixes ;

- traces semi-circulaires des canons enrouleurs;

- traces rectangulaires des rampes oscillantes (rares).

\section{Critères radiométriques}

Ils constituent des indices probables liés à des variations de la réflectance spectrale, soit du sol, soit du couvert végétal après application d'une dose d'arrosage.

\section{Réflectance spectrale du couvert végétal}

Les espèces végétales possèdent des courbes de réflectance spectrale présentant un maximum de réflexion pour des longueurs d'ondes comprises entre 700 et $1300 \mathrm{~nm}$ (Fig. 1). De nombreux travaux comme ceux de H.W. Gausman [1] ont montré que la réflectance spectrale des végétaux est essentiellement due à la réflexion de la lumière au contact des membranes cellulaires des tissus végétaux; la valeur de cette réflectance est fonction de la quantité de vides intercellulaires. En conséquence, toutes modifications de la quantité de ces vides et toute altération de la structure des tissus entrainnent des modifications des valeurs de la réflectance. a) Modifications des courbes de réflectance spectrale des végétaux

Les facteurs entraînant des modifications des courbes de réflectance spectrale sont, soit liés à la nature de la plante, soit extérieurs.

(i) facteurs liés à la nature de la plante :

- la structure du mésophylle qui peut être compacte ou vacuolaire et qui dans un premier cas entraîne un accroissement de la valeur de la réflectance ;

- la maturité de la plante qui accroît également la valeur de la réflectance ;

- la pigmentation par les chloroplastes, le carotène ou l'anthocyanine modifie l'absorption de la lumière et parallèlement la valeur de la réflectance qui diminue avec l'existence de pigments colorés (Fig. 2).

(ii) facteurs externes

- la déshydratation des tissus augmente la valeur de la réflectance(Fig. 3), mesure de laboratoire effectuée perpendiculairement à la feuille,

- la présence de fertilisants [2];

- l'existence de maladies comme la déficience en minéraux [3].

- ces facteurs ont une influence plus ou moins grande selon les conditions d'expériences, mais qui reste très en deçà de celle liée à la présence d'eau dans la plante, notamment en période de déficit hydrique. En effet, on constate que la réflectance totale augmente

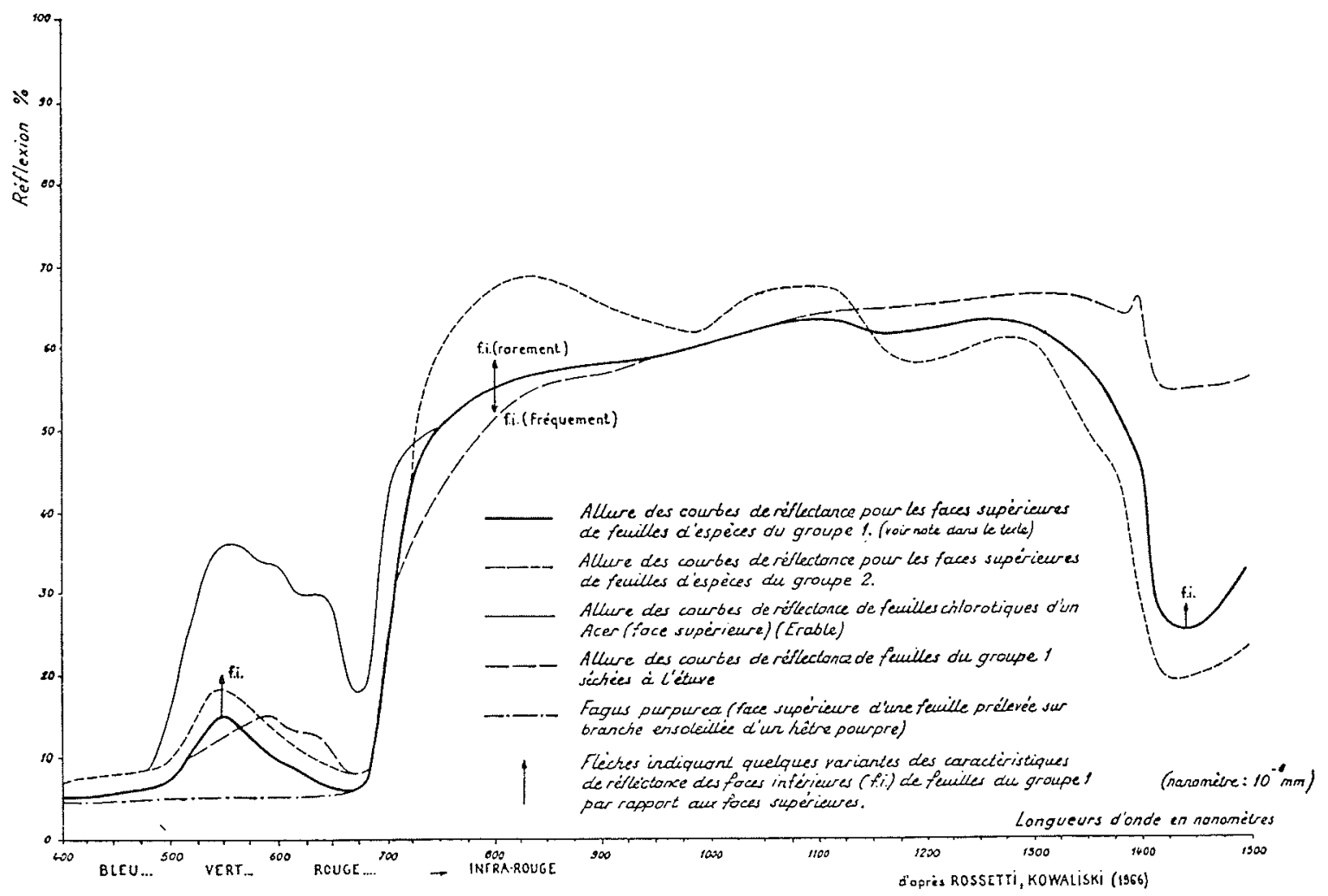

Figure 1-Quelques types de courbes de réflectance de plantes spontanées (domaine spectral : 400 à 1500 nanomètres). 


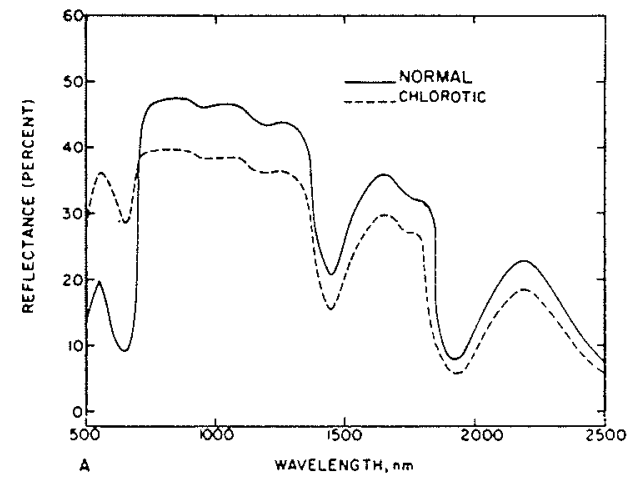

Figure 2. - Effet de l'augmentation de la teneur en chlorophylle sur la réflectance des feuilles (exemple pris sur la face supérieure de la feuille de sorgho)

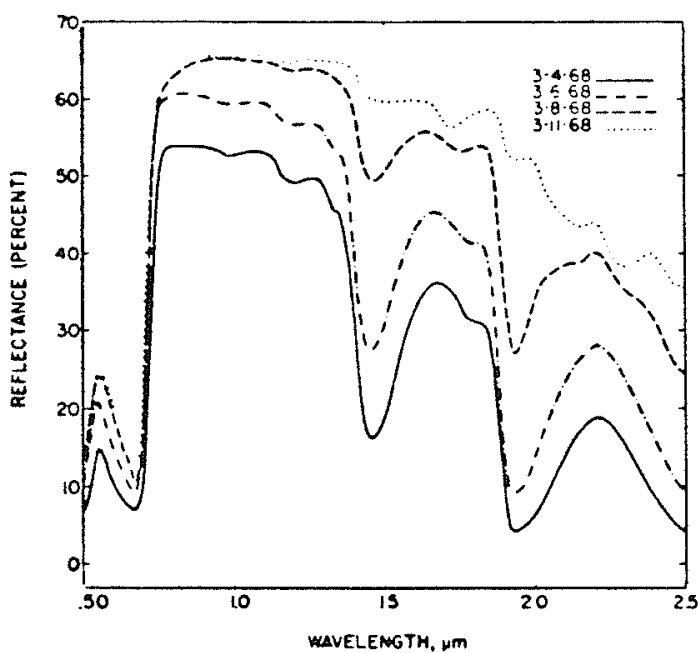

Figure 3 - Effet d'une déshydratation progressive sur la réflectance des faces supérieures des feuilles de coton. La courbe inférieure (trait plein) représente les feuilles fraîches et la courbe supérieure (pointillés) représente les feuilles après 7 jours de déshydratation.

lorsque les tissus sont turgescents, c'est à dire lorsque la plante est correctement alimentée en eau.

Une bonne alimentation en eau maintient l'activité végétative maximale surtout si l'on se situe dans une période de déficit hydrique où, sans apport artificiel d'eau,les plantes réduisent leur activité pour s'adapter aux conditions climatiques. La variation de la réflectance en fonction de la turgescence est surtout sensible en infra-rouge (Fig. 4).

La détection des parcelles irriguées s'est donc appuyée sur ces deux dernières propriétés (Photo 1).

\section{Réflectance spectrale du sol}

La réflectance spectrale du sol diminue avec la présence d'eau alors que le sol a naturellement une bonne réflexion dans le rouge et le proche infra-rouge (Photo 2).

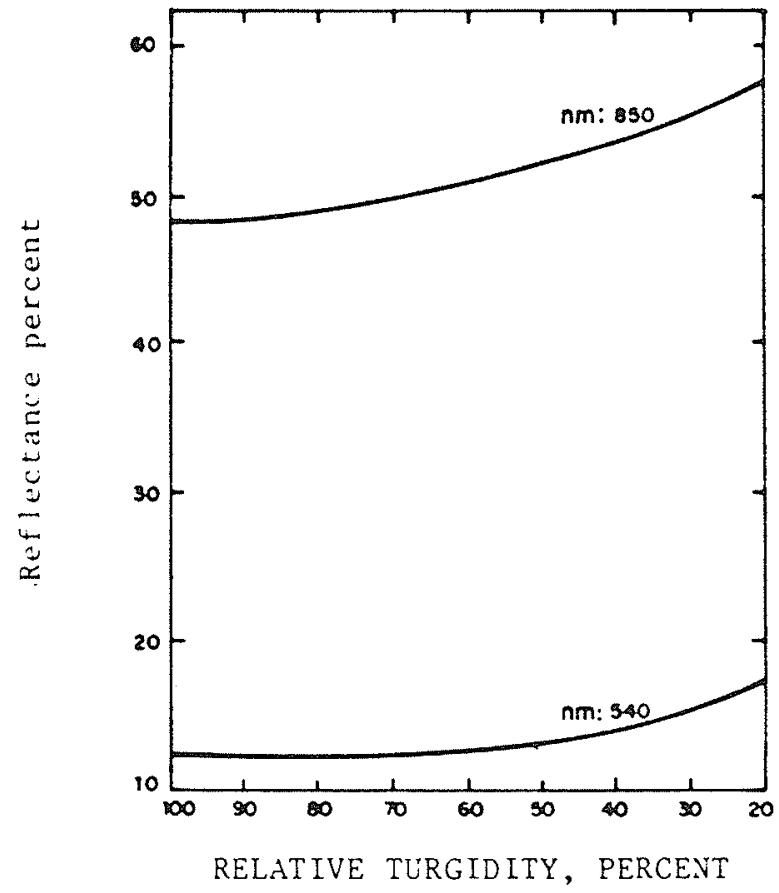

Figure 4 - Effet de la turgescence relative d'une feuille de coton sur les valeurs des longueurs d'onde données (ici l'infra-rouge $850 \mathrm{~nm}$ et le vert $540 \mathrm{~nm}$ ). Mesure de laboratoire effectuée perpendiculairement à la feuille.

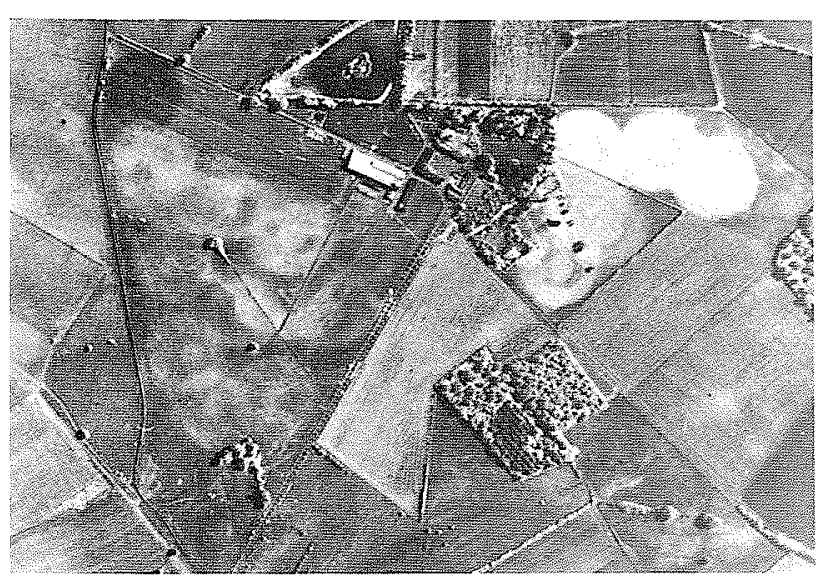

Photo 1

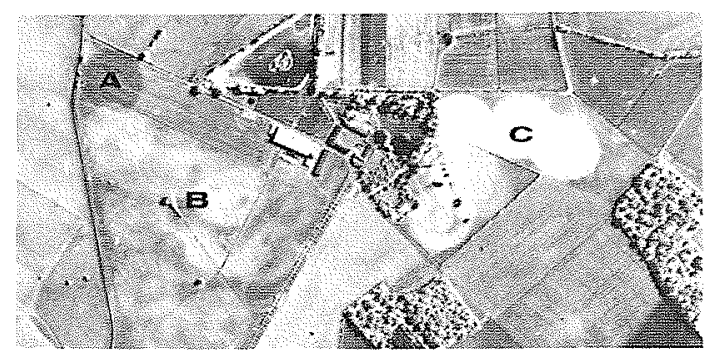

Photo 2 - Agrandissement (1/10 000 environ) d'un positif I.R. A - Trace sombre : distribution circulaire de l'eau sur sol visible (abaissement de la réflectance).

B - Montée en pression osmotique des plants irrigués.

C - Pression osmotique forte sur plants bénéficiant d'apport d'eau en période de déficit hydrique du sol (été 1976). 


\section{Identification photographique de l'irrigation}

L'utilisation des critères radiométriques doit s'entourer de certaines précautions; en effet :

a) une forte réflectance des végétaux et une faible réflectance du sol ne sont pas forcément le fait d'une irrigation mais peuvent entre autre traduire une forte rétention d'eau du sol ;

b) l'estimation de la valeur absolue de la réflectance est insuffisante, il est nécessaire d'utiliser la notion de réflectance différentielle :

- comparaison pour une même nature de culture entre parcelles voisines, l'une irriguée, l'autre non ;

- comparaison à l'intérieur d'une même parcelle sur deux zones inégalement irriguées.

Il est donc évident qu'outre les critères d'identification géométriques ou radiométriques, la détection des parcelles irriguées s'appuie sur le raisonnement traditionnel de la photo-interprétation : reconnaissance des principaux types culturels.

\section{L'opération "Télédétection irrigants 1976"}

L'opération "Télédétection Irrigants 1976" a comporté deux parties distinctes mais complémentaires :

- l'opération principale menée pour le compte de l'Agence par SETEC International,
- l'opération associée menée par l'ENITH pour le compte de la SADRAL (Société d'Aménagement de la Région Authion-Loire).

\section{L'opération Agence}

L'opération Agence s'est déroulée en quatre phases successives couvrant un périmètre de 2200000 ha dont une partie sur le Bassin "Seine-Normandie": (Fig. 5)

- Prises de vues aériennes

- Photo-interprétation

- Enquête cadastrale

- Contrôle et Vérité Terrain

\section{Les moyens photo-aériens}

Exception faite de la mission réalisée en 1975 par l'IGN pour le compte de l'Inventaire Forestier National, les prises de vues ont été réalisées par le Cabinet Rollin Gallot de Marseille, avec une caméra WILD RC 8 à focale de $152 \mathrm{~mm}$ munie d'un filtre adapté, embarquée à bord d'un Piper Aztêque volant à une altitude de $4500 \mathrm{~m}$.

L'émulsion retenue a été l'infra-rouge noir et blanc.

L'échelle choisie $1 / 30000$ répond aux exigences suivantes :

- adaptation à la taille du parcellaire moyen ;

- lisibilité aisée des indices géométriques :

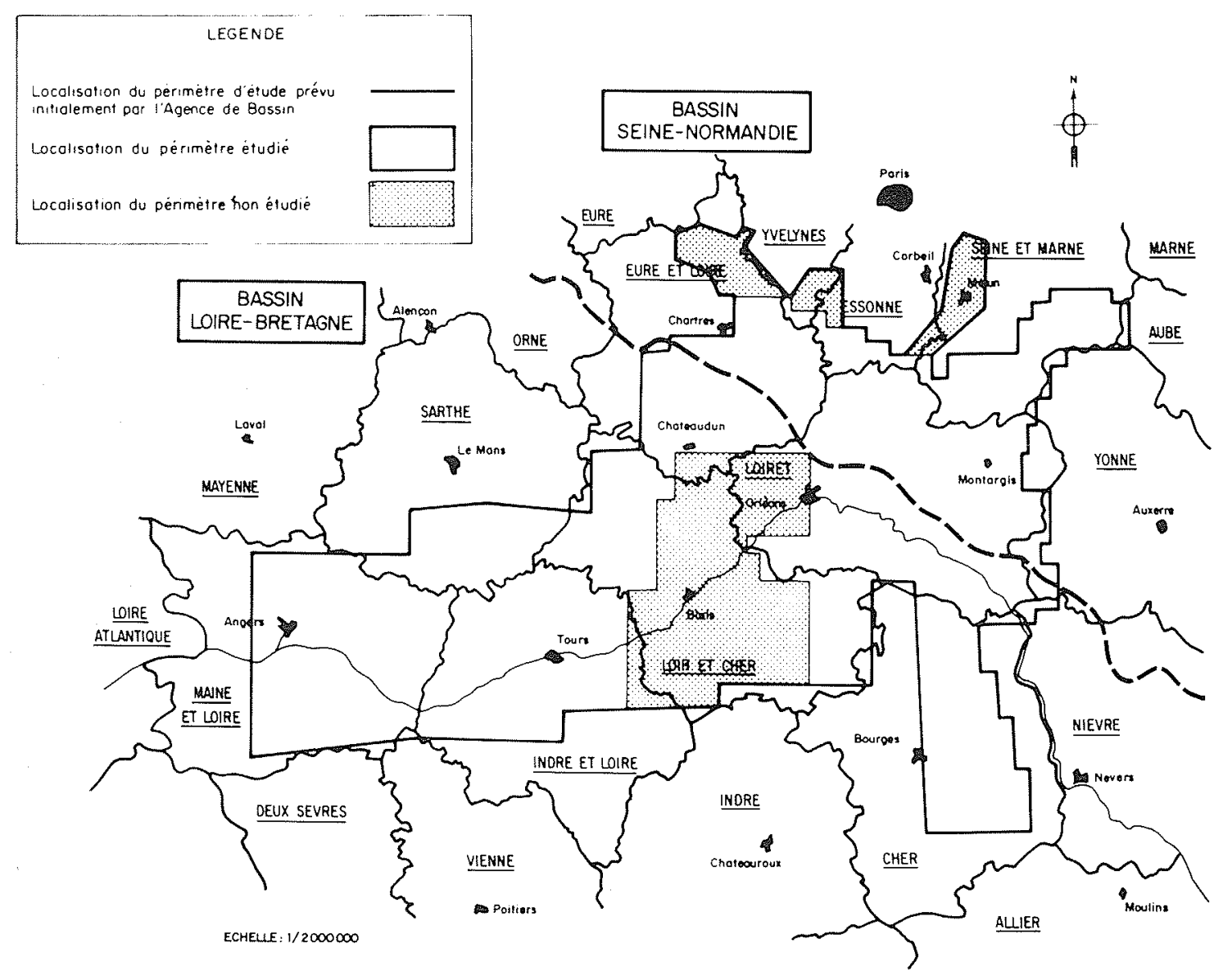

Figure 5 - Localisation du périmètre étudié 
- optimisation des moyens aériens en fonction de la surface à traiter ;

La durée de la couverture aérienne était estimée à 2 mois.

\section{Le déclenchement des prises de vues}

Pour que la détection aérienne des parcelles culturales irriguées soit possible, il est nécessaire que :

- les prises de vues soient effectuées dans une période de déficit hydrique des sols ;

- l'irrigation soit effective.

Dans la zone étudiée l'évolution des réserves en eau du sol a été suivie à partir de l'évapo-transpiration réelle mais et des pluies observées dans les stations météo de Angers, Bourges, Le Mans, Orléans et Tours.

La figure 6 présente pour Bourges et Orléans à partir du $1^{\text {er }}$ juin les précipitations observées et le déficit moyen journalier $(E T R-P)$ sur le mois calendaire.

La date des prises de vues est matérialisée par une flèche. Les mesures effectuées au sol ont permis de considérer que la réserve facilement utilisable était annulée dès le 8 juillet en 1975, dès le 11 Juin en 1978

Les irrigations ont été déclenchées à ces dates par les exploitants, le dernier tour d'eau se situant au 10 août.

Les perturbations orageuses observées en juillet 1976 ont donc provoqué un retard dans les prises de vues de 3 semaines qui a conduit à prendre un certain risque dans le déclenchement des premières prises de vues (23 juillet).
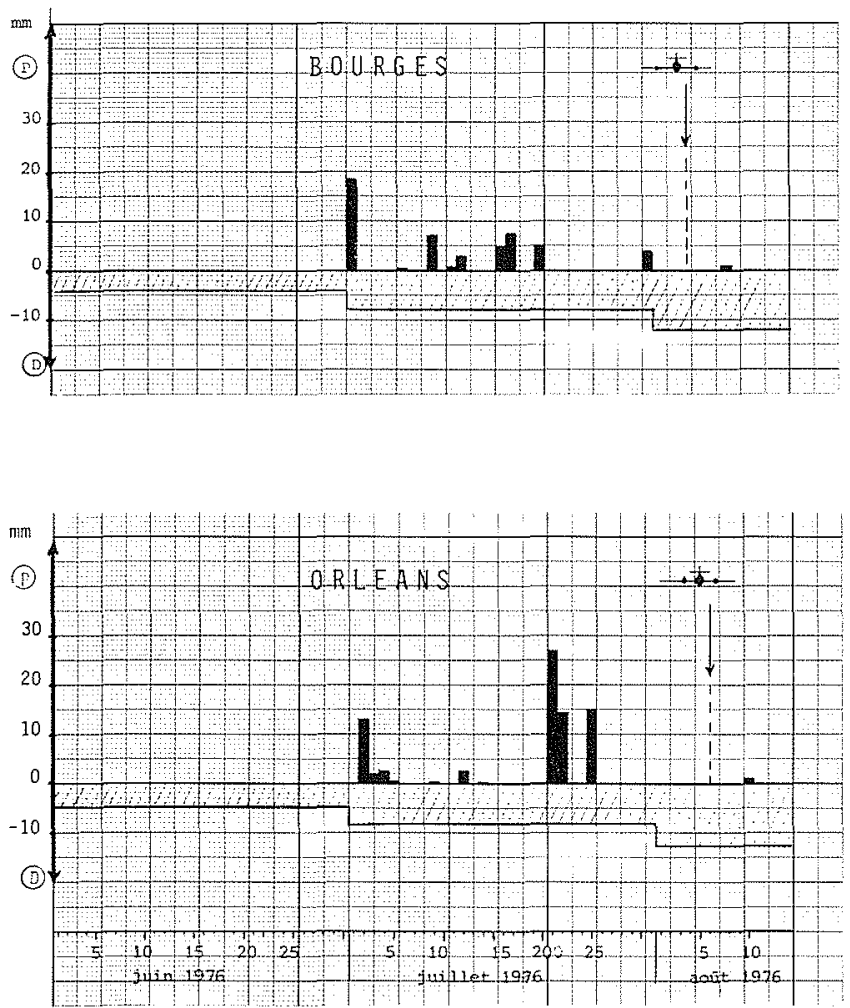

Figure 6 - Evolution des ressources en eau

$P$ : Précipitations en $\mathrm{mm}$

$D$ : Déficit hydrique $(E T R-P)$ journalier en $\mathrm{mm}$.

\section{Photo-interprétation}

L'interprétation des images obtenues s'est effectuée directement sur clichés

- contacts papier dans le cas des missions IGN IFN

- négatifs pour les missions aériennes effectuées en 1978.

Les informations ont été notées sur calques superposés aux clichés après interprétation sous stéréoscope et table lumineuse pour les négatifs.

L'interprétation des clichés à conduit à classer les parcelles détectées de la manière suivante :

- Présence simultanée des critères géométriques et radiométriques : surement irrigué : 10

- Présence des seuls critères radiométriques : probablement irrigué : 20

- Irrigation difficilement décelable du fait de la taille de la parcelle : 30

Les mosaïques d'assemblage ont ensuite été équipées des limites administratives des communes. Cette phase a duré 6 mois.

\section{Enquête cadastrale}

L'enquête a dû être menée dans les locaux du cadastre sur les minutes de conservation (seuls documents à jour), afin d'identifier les parcelles cadastrales concernées par la localisation des parcelles culturales détectées.

Une parcelle culturale peut en effet ètre intégralement comprise dans une parcelle cadastrale ou couvrir tout ou partie de deux, voire trois parcelles cadastrales. Cette enquête s'est terminée avec la prise de contact des propriétaires des parcelles concernées.

\section{L'opération SADRAL}

Cette opération avait pour but de comparer les surfaces détectées avec un relevé au sol et d'étudier l'intérêt de l'émulsion de l'infra-rouge couleur dans l'observation des zones irriguées.

Les prises de vues ont eu lieu le 22 juillet avec un appareil Hasselblad $6 \times 6$ équipé d'un moteur électrique commandé par intervallomètre monté sur un avion léger du $3^{\mathrm{e}}$ GALREG mis à disposition par les autorités militaires.

L'objectif était équipé d'un filtre jaune Kodak Wratten 25 A pour pouvoir travailler avec une émulsion infra-rouge couleur.

Le plan de vol et le découpage du parcellaire dans la zone étudiée ont conduit à retenir l'échelle du 1/13000.

Le périmètre retenu se situe en Anjou dans la vallée de 1'Authion sur trois communes (Brain sur Authion, La Daguenière, La Bohalle) où les sols sont différents :

- Sableux à sablolimoneux à Brain

- Sabloargileux dans les deux autres communes.

La zone retenue était parcourue selon un circuit préétabli par un observateur qui notait pour les parcelles en cours d'arrosage :

- La parcelle arrosée

- Le type d'arroseur

- L'espèce arrosée. 
Pour des raisons matérielles, ces observations au sol étaient limitées au matin de $8 \mathrm{~h}$ à $12 \mathrm{~h}$ et se déroulèrent durant les deux semaines précédant le vol,exception faite des 14 et 21 juillet.

\section{Résultats de l'opération "Télédétection Irrigants 1976". Analyse et commentaire}

Globalement les résultats de l'opération "Télédétection Irrigants 1976 " pour l'ensemble de la zone étudiée située dans le Bassin "Loire-Bretagne" se présentent de la façon suivante :

\begin{tabular}{|c|c|c|}
\hline Irrigation & $\begin{array}{c}\text { Superficie } \\
\text { détectée }\end{array}$ & $\begin{array}{c}\text { Nombre de } \\
\text { parcelles }\end{array}$ \\
\hline Sure & 27900 ha & 22800 \\
\hline Probable & 98700 ha & 54400 \\
\hline $\begin{array}{c}\text { Difficile à } \\
\text { déceler/taille }\end{array}$ & 1400 ha & 590 \\
\hline
\end{tabular}

La taille des parcelles détectées varie de 0,6 ha dans le Maine et Loire (maraîchage, pépinières) à 7 ha dans l'Eure et Loir (grande culture).

Le tableau 1 donne le détail de ces valeurs par département.

A titre d'information le nombre des propriétaires concernés est de 39000 au total dont 33000 avec moins de 6 hectares détectés.

Analyse et comparaisons des résultats 1976 avec les données du Recensement Général de l'Agriculture de 1976

La forte proportion représentée par les parcelles classées en "Irrigation probable" ne semblait pas poser problème au début de l'exploitation de l'enquête, compte tenu des circonstances climatiques particulières de 1976. Cependant, après plusieurs réponses négatives et controles sur le terrain, une analyse comparée des résultats au niveau des cantons ou des régions agricoles s'est révélée nécessaire.

Le tableau 2 présente par régions agricoles les résultats de 1976 et ceux du RGA 1970.

On constate que les valeurs importantes du rapport des superficies irriguées probables 1976 sur irrigué du RGA 1970 correspondent à des régions agricoles à hydromorphie marquée, soit qu'il s'agisse de sols lourds à forte teneur en argile, soit de terrain difficile à assainir (Sologne, Perche Vendomois, Pays fort et Sancerrois, Faux Perche et Grande Sologne).

Il semble donc que la probabilité de l'irrigation soit beaucoup plus discutable que ne le laissaient supposer les premiers points de vue, le bon état végétatif traduit par la réflectance spectrale forte étant à attribuer à 1'hydromorphie, favorable à la végétation en 1976, et non à l'irrigation.

\begin{tabular}{|c|c|c|c|}
\hline \multirow{2}{*}{$\begin{array}{l}\text { Tableau } 1 \\
\text { Département }\end{array}$} & \multicolumn{3}{|c|}{ Superficies détectées par département } \\
\hline & Catégorie & $\begin{array}{l}\text { Surface } \\
\text { totale }\end{array}$ & $\begin{array}{l}\text { Nombre de } \\
\text { parcelles }\end{array}$ \\
\hline \multirow{3}{*}{18} & 10 & 2576 & 364 \\
\hline & 20 & 14004 & 3366 \\
\hline & 30 & 35 & 19 \\
\hline \multirow{3}{*}{28} & 10 & 4284 & 1712 \\
\hline & 20 & 11756 & 6918 \\
\hline & 30 & 6 & 7 \\
\hline \multirow{3}{*}{37} & 10 & 1836 & 3036 \\
\hline & 20 & 7976 & 6993 \\
\hline & 30 & 5 & 24 \\
\hline \multirow{3}{*}{41} & 10 & 2510 & 965 \\
\hline & 20 & 11567 & 5041 \\
\hline & 30 & 4 & 2 \\
\hline \multirow{3}{*}{45} & 10 & 8257 & 3563 \\
\hline & 20 & 33933 & 11617 \\
\hline & 30 & 1341 & 524 \\
\hline \multirow{3}{*}{49} & 10 & 7850 & 12767 \\
\hline & 20 & 13898 & 15511 \\
\hline & 30 & 5 & 11 \\
\hline \multirow{3}{*}{72} & 10 & 565 & 363 \\
\hline & 20 & 5515 & 4968 \\
\hline & 30 & - & - \\
\hline \multirow{3}{*}{$\begin{array}{l}\text { Total "Loire- } \\
\text { Bretagne" }\end{array}$} & 10 & 27878 & 22770 \\
\hline & 20 & 98653 & 54412 \\
\hline & 30 & 1396 & 587 \\
\hline \multicolumn{4}{|c|}{$\begin{array}{l}10 \text { - Sûrement irrigué } \\
20 \text { - Probablement irrigué } \\
30 \text { - Irrigation difficilement décelable du fait de la taille de la } \\
\text { parcelle. }\end{array}$} \\
\hline
\end{tabular}

\section{Les résultats de l'opération SADRAL}

L'expérience a montré tout d'abord la précarité d'un repérage au sol qui nécessiterait une observation de jour comme de nuit et, pendant un minimum de quinze jours, un nombre important d'observateurs.

En effet pendant la phase d'observation, 172 ha ont été repérés au sol, alors que la télédétection a décelé 361 ha "Irrigation Sure" confirmés par la SETEC et l'ENITH.

Une seconde conclusion portant sur la rémanence des traces de l'irrigation a pu être apportée :

- En sol nu, la rémanence de l'irrigation ne dépasse pas 3 jours quel que soit le type de sol;

- Sur la végétation la rémanence de l'irrigation pourrait atteindre 14 jours. 


\begin{tabular}{|c|c|c|c|c|c|c|}
\hline \multicolumn{2}{|l|}{ Tableau 2} & \multicolumn{5}{|c|}{ Comparaison des résultats Télédétection 76 - RGA 70 par Régions Agricoles } \\
\hline Région Agricole & R.G.A. & Agence & $\begin{array}{l}\text { Irrigué } \\
10\end{array}$ & $\begin{array}{l}\text { Irrigué } \\
10+20\end{array}$ & $\begin{array}{c}\text { Probable } \\
\text { R.G.A. } \\
\%\end{array}$ & $\begin{array}{c}\text { Date du } \\
\text { vol }\end{array}$ \\
\hline Beauce & 9126 & 7616 & 3161 & 13937 & 1,01 & $\begin{array}{c}13.7 .75 \text { et } \\
5.8 .76\end{array}$ \\
\hline Faux Perche & 101 & 72 & 46 & 758 & 7,05 & 13.7 .75 \\
\hline $\begin{array}{l}\text { Champagne } \\
\text { Berrichonne }\end{array}$ & 856 & 965 & 1400 & 5171 & 4,04 & 4.8 .76 \\
\hline Beaujois & 1268 & 471 & 967 & 3628 & 2,10 & 25.7 .76 \\
\hline Vallée du Loir & 1161 & 609 & 280 & 932 & 0,56 & 26.7 .76 \\
\hline $\begin{array}{l}\text { Vallée de la } \\
\text { Sarthe et } \\
\text { Région mancelle }\end{array}$ & 233 & 35 & 23 & 1058 & 4,44 & 26.7 .76 \\
\hline Gatine Tourangelle & 935 & 485 & 286 & 3814 & 3,77 & 24.7 .76 \\
\hline Champeigne & 333 & 242 & 224 & 865 & 1,92 & 24.7 .76 \\
\hline Val de Loire & 857 & 308 & 748 & 2217 & 1,71 & 26.7 .76 \\
\hline $\begin{array}{l}\text { Région Viticole } \\
\text { à l'Est de Tours }\end{array}$ & 250 & 209 & 45 & 655 & 2,44 & 24.7 .76 \\
\hline Sologne & 180 & 486 & 651 & 6470 & 32,33 & 27.7 .75 \\
\hline Grande Sologne & 212 & 374 & 409 & 1818 & 6,65 & 6.8 .76 \\
\hline Saumurois & 470 & 359 & 372 & 2190 & 3,87 & 25.7 .76 \\
\hline Choletais & 1010 & 564 & 569 & 2945 & 2,35 & 23.7 .76 \\
\hline Beauce Dunoise & 10942 & 8241 & 2046 & 5291 & 30 & 4.8 .75 \\
\hline $\begin{array}{l}\text { Vallée et coteaux } \\
\text { du Loir }\end{array}$ & 433 & 289 & 339 & 2509 & 5,01 & 5.8 .76 \\
\hline Perche Vendomois & 87 & 39 & 56 & 1407 & 15,52 & 5.8 .76 \\
\hline $\begin{array}{l}\text { Pays Fort et } \\
\text { Sancerrois }\end{array}$ & 132 & 178 & 205 & 1907 & 12,89 & 4.8 .76 \\
\hline
\end{tabular}

\section{Conclusion - Perspectives}

Les résultats obtenus, les diverses photos présentées montrent que la détection des parcelles irriguées en complément dans les circonstances climatologiques de la Région Centre ou Pays de la Loire, est possible et relativement facile. Cependant pour obtenir un résultat exhaustif, cette recherche doit s'appuyer sur deux séries de clichés décalés d'un demi-tour d'eau soit de 4 à 6 jours.

Les conditions initiales étant particulièrement importantes, il est nécessaire de retarder d'au moins une semaine la prise de vue après une précipitation dépassant 40 millimètres.
Dans ces conditions, le repérage des parcelles peut être assuré car les critères géométriques, à défaut de connaissance précise de la Réserve utile des sols sur le terrain objectif, constituent la preuve indiscutable d'une irrigation.

Le coût de cette seconde prise de vue et de son interprétation ne constitue pas une charge supplémentaire car en fait la précision de l'interprétation est đe nature à réduire le temps consacré à l'enquête cadastrale et à la phase administrative de la recherche.

En ce qui concerne l'échelle des prises de vue, il semble préférable sur de vastes régions d'envisager d'adapter l'échelle au parcellaire, le 1/50000 en Beauce est acceptable, alors que le 1/25000 dans le Maine et Loire est nécessaire. 
Pour conclure sur des orientations possibles permettant de minimiser les coûts de cette recherche, il nous semble que l'exploitation des données fournies par les satellites du type Landsat ou des nouvelles générations devrait permettre du fait de la fréquence des passages de localiser plus nettement et plus facilement les zones où l'irrigation se pratique, réduisant de ce fait le champ d'investigation des vecteurs aériens pour déterminer les parcelles concernées.

Par ailleurs, ces exploitations de données satellites permettront de saisir l'évolution des zones irriguées et par là même de déterminer le seuil de rentabilité d'une recherche complémentaire.

\section{Bibliographie}

[1] GAUSMAN H.W. - Leaf reflectance of near infrared Photogrammetric Engineering, vol. $\mathrm{XL}, \mathrm{N}^{\circ} 2,1974$.

[2] THOMAS J.R., GERBERMANN A.H. - Yield reflectance relations in cabbage - Photogrammetric Engineering, vol 43 $n^{\circ} 10-1977$.

[3] GAUSMAN H., CARDENAS R., GERBERMANN A.M. Plant size, etc, and aerial films Photogrammetric Engineering, vol XL n ${ }^{\circ} 1-1974$

\section{Discussion}

Le Président remercie les auteurs de l'expérience pratique fort intéressante dont ils ont fait part.

M. GHIO observe qu'il convient d'être très prudent sur l'interprétation des résultats et que les écarts mentionnés de 1 à 4 entre données RGA et superficies détectées - sûres ou probables - sont peut être excessifs.

M. ROMAN précise que les enquêtes faites entre 1970 et 1976 laissent penser que, suivant cette période, l'augmentation des surfaces irriguées a été de l'ordre de 40 à $50 \%$.

Une remarque de M. RODIER sur l'intérêt des contrôles au sol au cours de l'opération amène $M$. ROMAN à citer quelques exemples précis illustrant la difficulté d'interprétation des données recueillies et les déconvenues auxquelles cela a parfois conduit.

Finalement, conclut-ii, je ne pense pas que le problème essentiel ait été, en 1976, de multiplier les contrôles au sol; nous nous orientons plutôt vers la double prise de vue en s'en tenant aux critères géométriques

Le Président demande à M. ROMAN quelle a été la ligne de conduite adoptée concernant l'exploitation administrative
- pour l'assiette de la redevance, notamment-des résultats recueillis.

M. ROMAN précise que cette exploitation n'a pas fait l'objet d'investigation draconnienne ou policière, mais était fondée sur une concertation très ouverte tant avec les propriétaires concernés qu'avec l'ensemble de la profession "qui est consciente et demanderesse concernant le droit à l'eau et qui sait que le droit à l'eau a pour contrepartie un minimum de devoirs, le premier étant de déclarer son activité, le second de payer la redevance...".

Mme VALERIO remarque que les écarts de réflectance constatés suivant que l'on opérait dans l'infra-rouge ou à $850 \mathrm{~nm}$ ne lui paraisaient pas avoir été exploités au cours des expériences. $\mathrm{Ne}$ serait-il pas possible, poursuit-elle, d'améliorer la méthode en utilisant deux longueurs d'onde au lieu d'une seule et en combinant l'interprétation des observations?

M. ALMAYRAC remarque que si de nombreux facteurs interviennent dans la variation de réflectance, l'eau demeure de beaucoup le plus important, souvent liée à des écarts relatifs de 40 à $50 \%$. II pense comme Mme VALERIO que l'exploitation combinée de deux longueurs d'onde serait peut être porteuse de résultats plus intéressants. 